\title{
Psychiatrische Praxis Jahrgang 34: Bewährtes und Neues
}

Im nunmehr 34. Jahrgang der Psychiatrischen Praxis werden Sie, liebe Leser, nicht nur bewährte Inhalte und Rubriken wiederfinden, sondern auch auf einige Neuerungen stoßen. Das betrifft nicht nur das Titelbild, sondern auch das gesamte Layout unserer Zeitschrift. Der Thieme-Verlag hat all seine Zeitschriften neu gestaltet. Die Veränderungen zielen auf eine größere Übersichtlichkeit der Beiträge und eine bessere Orientierung im Heft und sollen Ihre Freude und den fachlichen Gewinn bei der Lektüre der Zeitschrift erhöhen.

Wahrscheinlich haben Sie beim Blick auf die Titelseite des aktuellen Hefts schon gesehen, dass die Psychiatrische Praxis ab dem Jahr 2007 mit der ebenfalls bei Thieme verlegten Zeitschrift Krankenhauspsychiatrie, die Ende 2006 das letzte Mal erschienen ist, fusionieren wird. Wir freuen uns über die Erweiterung unseres Leserkreises und hoffen, dass die Themen der Psychiatrischen Praxis auch unsere neuen Leser ansprechen werden. Im Zusammenhang mit der Fusion, die ein Jahr lang auf der Titelseite unserer Zeitschrift vermerkt sein wird, wurde Tilman Steinert, der bislang die Krankenhauspsychiatrie mitgestaltet hat, in den Herausgeberkreis der Psychiatrischen Praxis berufen. Er wird in Zukunft gemeinsam mit Ulrike Hoffmann-Richter und Philipp Portwich den Szene-Teil unserer Zeitschrift redaktionell betreuen. Federführend im Herausgeberkreis ist ab dem Jahr 2007 Steffi Riedel-Heller. Matthias Angermeyer scheidet zeitgleich mit seiner Emeritierung aus dem aktiven Herausgeberkreis aus. Wir danken ihm für sein langjähriges Engagement für die Psychiatrische Praxis und blicken an anderer Stelle in diesem Heft noch einmal auf seine wissenschaftliche Arbeit zurück.

Neben der seit einem Jahr bestehenden erfolgreichen Zusammenarbeit mit der Deutschen Gesellschaft für Gerontopsychiatrie und -psychotherapie (DGGPP) wird die Psychiatrische Praxis ab dem Jahr 2007 auch zum Organ des Arbeitskreises der Chefärzte und Chefärztinnen von Kliniken für Psychiatrie und Psychotherapie an Allgemeinkrankenhäusern in Deutschland (ACKPA) und der Bundesdirektorenkonferenz Deutscher Kliniken für Psychiatrie und Psychotherapie (BDK). Wir freuen uns sehr über diese beiden neuen Organschaften und sind uns sicher, dass gerade diese Kombination interessante und auch kontroverse Diskussionen entfacht.

Trotz der vielen Neuerungen wird die Psychiatrische Praxis auch in ihrem 34. Jahrgang auf bewährte Inhalte setzen. Dazu gehört die noch recht junge Tradition, am Anfang eines Jahres die drei meistzitierten Beiträge der Zeitschrift zu würdigen. Diesmal bezieht sich die Auswahl auf alle im Jahr 2004 (Jahrgang 31) publizierten Arbeiten.

Auf Platz drei liegt eine Untersuchung von Maria Fischer et al. zur Lebensqualität von Angehörigen schizophren Erkrankter. Die Studie zeigt, dass Furcht vor Diskriminierung, Sorge um die spätere Betreuung des Erkrankten und das Gefühl einer Sicherheitsbeeinträchtigung bei Angehörigen schizophren Erkrankter [1] mit einer geringeren Lebensqualität einhergehen. Die Psychologin und Psychotherapeutin Maria Fischer leitet die Innsbrucker Regionalgruppe des Angehörigenvereins „Hilfe für Angehörige Psychisch Erkrankter (HPE)“.

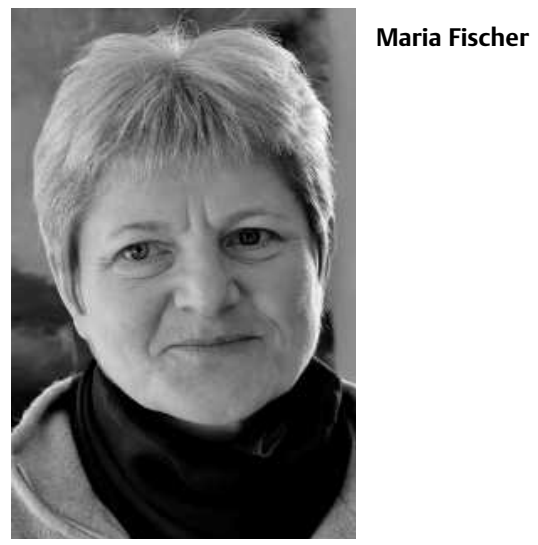

Auf Platz zwei liegt eine Arbeit, die in Kooperation zwischen der Psychiatrischen Universitätsklinik Tübingen und der Justitzvollzugsanstalt Rottenburg entstanden ist und einen Überblick über den Bedarf an psychiatrischer Mitbetreuung in Justizvollzugsanstalten gibt [2]. Der Erstautor, Gerd Schäfer, hat als Psychiater an der Universi-

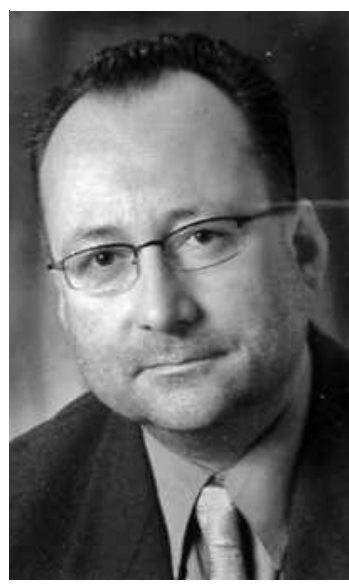

Gerd Schäfer

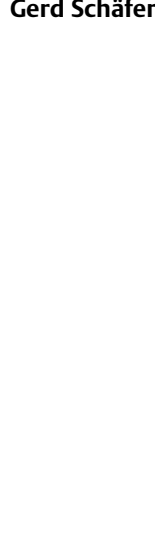

DOI 10.1055/s-2006-951939

Psychiat Prax 2007; 34: 2 -

Stuttgart · New York .

Korrespondenzadresse

Dr. med. Christiane Roick

Klinik für Psychiatrie,

Universität Leipzig

Johannisallee 20

04317 Leipzig

roich@medizin.uni-leipzig.de 
tätsklinik Tübingen gearbeitet und ist inzwischen in eigener Praxis tätig. In ihrem Beitrag weisen die Autoren um Gerd Schäfer einen hohen Bedarf an psychiatrischer Mitbetreuung in Justizvollzugsanstalten nach und plädieren dafür, dass auch in einem konsiliarischen Rahmen eine sinnvolle Therapie möglich ist.

Der am häufigsten zitierte Beitrag des Jahrgangs 31 basiert auf einer Studie, die im Kompetenznetz Depression und Suizidalität durchgeführt wurde. Der Erstautor des Artikels, Hans Joachim Salize, ist Soziologe und leitet die Arbeitsgruppe Versorgungsforschung am Zentralinstitut für Seelische Gesundheit in Mannheim. Die Arbeit von Salize et al. informiert über die Kosten der Versorgung depressiver Patienten in haus- und fachärztlicher Behandlung [3]. Sie weist auf eine erhebliche und kostenintensive somatische Komorbidität depressiv erkrankter Patienten hin.

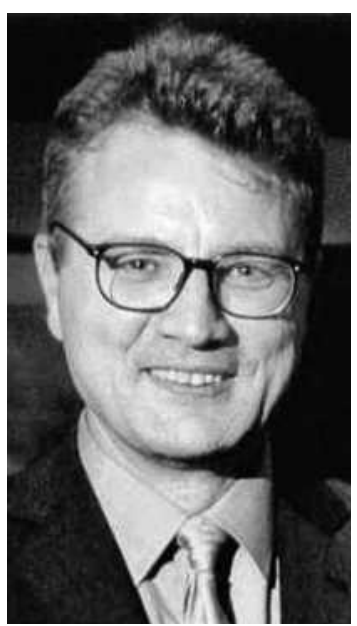

Hans Joachim Salize
Die Siegerarbeiten des Jahrgangs 31 spiegeln die ganze Breite des Themenspektrums der Psychiatrischen Praxis wider. Darüber hinaus sprechen die unterschiedlichen beruflichen Schwerpunkte der Erstautoren dafür, dass die Psychiatrische Praxis ein echtes Forum für interdisziplinäre Zusammenarbeit ist.

Wir gratulieren den Autoren der drei meistzitierten Arbeiten zu ihren Beiträgen, bedanken uns aber auch bei allen anderen Autoren und natürlich bei den Lesern, die dazu beigetragen haben, dass die Psychiatrische Praxis eine vielseitige Zeitschrift und ein Forum für den fachlichen Austausch zwischen allen an psychiatrischer Versorgung und Forschung interessierten und engagierten Berufsgruppen ist.

Christiane Roick

Steffi G. Riedel-Heller

\section{Literatur}

1 Fischer M, Kemmler G, Meise U: „Schön, dass sich auch einmal jemand für mich interessiert" - Eine Erhebung der Lebensqualitat von Angehörigen langzeitig an Schizophrenie Erkrankter. Psychiat Prax 2004; 31: $60-67$

2 Schäfer G, Schubert W, Bartels M, Foerster K: Psychiatrische Konsiliartätigkeit in der Justizvollzugsanstalt Rottenburg. Psychiat Prax 2004; 31: $4-10$

3 Salize HJ, Stamm K, Schubert M et al: Behandlungskosten von Patienten mit Depressionsdiagnose in haus- und fachärztlicher Versorgung in Deutschland. Psychiat Prax 2004; 31: 147 - 156 